\title{
Archipel
}

ARCHIPEL Études interdisciplinaires sur le monde insulindien

$93 \mid 2017$

Varia

\section{Continuités et transitions de la Reformasi indonésienne : l'actualité politique de Banten à l'issue des élections provinciales de 2017}

Continuities and transitions of the Indonesian Reformasi. Banten political news after the 2017 provincial elections

\section{Gabriel Facal}

\section{OpenEdition}

Journals

Édition électronique

URL : http://journals.openedition.org/archipel/410

DOI : 10.4000/archipel.410

ISSN : 2104-3655

Éditeur

Association Archipel

Édition imprimée

Date de publication : 6 juin 2017

Pagination : 133-150

ISBN : 978-2-910513-74-0

ISSN : 0044-8613

\section{Référence électronique}

Gabriel Facal, «Continuités et transitions de la Reformasi indonésienne : l'actualité politique de Banten à l'issue des élections provinciales de 2017 », Archipel [En ligne], 93 | 2017, mis en ligne le 01 juin 2017, consulté le 20 avril 2019. URL : http://journals.openedition.org/archipel/410; DOI : 10.4000/

archipel. 410 
GABRIEL FACAL *

\section{Continuités et transitions de la Reformasi indonésienne : l'actualité politique de Banten à l'issue des élections provinciales de 2017}

\section{Introduction}

L'actualité politique de la province de Banten, à Java Ouest, est marquée par l'incertitude quant au résultat des élections provinciales de février dernier, lesquelles ont opposé le binôme Rano Karno-Embay Mulya Syarif et l'alliance entre Wahidin Halim et Andika Hazrumy ${ }^{1}$. Le résultat (respectivement $49,05 \%$ contre $50,95 \%$ ) a été contesté par le premier binôme, lequel a porté l'affaire devant la Cour constitutionnelle (Mahkamah Konstitusi) en accusant l'alliance rivale d'avoir acheté les votes de milliers d'électeurs. En amont des élections, l'attention des commentateurs de la politique bantenoise avait surtout été retenue par l'opposition entre les deux candidats à la vice-gouvernance. Le premier, Andika Hazrumy, est le fils aîné de la gouverneure, Atut Chosiyah, emprisonnée pour corruption en décembre 2013, peu avant que soit écroué son frère cadet, Chaeri Wardana. Quant à Embay Mulya Syarif, il est l'ancien bras droit du père d'Atut Chosiyah, Chasan Sochib. Celui-ci n'occupa jamais de position politique formelle mais il fut le maître de l'échiquier politique bantenois durant une quarantaine d'années, à partir de la fin des années 1960 jusqu'à son décès en juin 2011.

Le colistier d'Andika Hazrumy, Wahidin Halim, fut maire de Tangerang entre 2003 et 2013, et il représenta le principal rival de la famille d'Atut *. Chercheur postdoctorant, associé à l'Institut de recherches Asiatiques (IrAsia).

1. Je souhaite remercier Monsieur Abdul Hamid (enseignant de sciences de l'administration publique à l'Universitas Tirtayasa, Serang, Indonésie), qui m'a transmis un nombre considérable d'informations ayant étayé cette étude de l'actualité politique bantenoise. 
Chosiyah dans cette circonscription. Quand au colistier d'Embay Mulya Syarif, Rano Karno, il fut initialement un opposant à la famille de Sochib durant les élections de 2008 pour la préfecture de Tangerang. Élu vice-préfet, il fut ensuite nommé en 2011 vice-gouverneur de Banten. À partir de 2013, il prit les fonctions de gouverneur par intérim pour remplacer Atut Chosiyah.

$\mathrm{Au}$ vu de leur volatilité, les coalitions des dernières élections ont surpris les analystes de la politique bantenoise ainsi que les journalistes de la presse régionale. En outre, la campagne électorale avait été précédée de rapprochements stratégiques entre Rano Karno et Khaerul Jaman (frère cadet d'Atut Chosiyah), puis Andika Hazrumy, laissant penser que la famille de Sochib continuait de dominer les jeux de coalition et la donne électorale. En définitive, bien que plusieurs membres de la famille soient parvenus à conserver des fonctions politiques importantes, la disparition ou l'éviction des figures dominantes que représentaient Sochib, Atut Chosiyah et Chaeri Wardana, semblent avoir ébranlé la cohésion familiale. Par conséquent, on peut se demander si les réseaux familiaux développés par Sochib parviendront à l'emporter aux élections. Et si Hazrumy essuie une défaite, on peut s'interroger sur la capacité de la famille à maintenir ses monopoles économiques et politiques.

Pour aborder cette question, j'établirai ici un état des lieux des évolutions politiques bantenoises récentes ${ }^{2}$. Il permettra d'analyser les enjeux politiques qui se posent à l'issue des élections provinciales pour Banten et ses dix millions d'habitants. Comme l'a souligné Paul Carnegie (2009 : 521), l'examen des interactions entre l'action politique et les institutions peut permettre de repérer les contrastes des dynamiques politiques locales dans le cadre du processus de démocratisation indonésien. Dans le cas spécifique de Banten, l'étude de la décentralisation suggère aussi que pour décoder les évolutions politiques actuelles et leurs enjeux il est nécessaire de considérer les dynamiques régionales au travers d'une perspective diachronique, d'examiner les relations interpersonnelles qui lient les acteurs politiques de la province et d'étudier les composantes nationales qui exercent une pression sur les dynamiques régionales.

\section{Le parcours politique d'Atut Chosiyah et de sa famille}

Les conditions de concentration du pouvoir politique par la famille de Sochib sont liées aux activités que celui-ci a initialement développées dans le secteur de la petite criminalité. À la fin des années 1950, il s'initia au commerce en reprenant le négoce de denrées alimentaires de son père, puis se forma progressivement aux techniques de gestion commerciale, tout

2. Je me baserai sur la dizaine de terrains (trente-sept mois entre 2004 et 2017) que j'ai réalisés en Indonésie et plus particulièrement dans la région de Banten. J'actualiserai ainsi les travaux que j'ai menés par le passé sur les dynamiques politiques bantenoises (Facal 2012 ; 2014). 
d'abord en important ses produits depuis Lampung (au sud de Sumatra) et en les revendant à Jakarta, puis en diversifiant ses activités, à grand renfort d'intimidations et de représailles. Dès 1967, il prit la direction de l'entreprise de construction Andalas, puis en 1969 celle de Sinar Ciomas, grâce à laquelle il fit fortune et qui reste aujourd'hui une entreprise majeure du secteur de la construction au niveau régional. Dès ses débuts en affaires il entretint une collaboration forte avec Embay Mulya Syarif. Celui-ci bénéficiait de soutiens au sein de l'aile islamique de l'armée (son père était lui-même officier d'une division à Serang) et venait ainsi faire contrepoids à la tutelle militaire nationaliste qui pesait sur les entreprises de Sochib.

Pour mener à bien leurs affaires, les deux associés développèrent un réseau clientélaire au sein de l'univers des " hommes forts », localement appelés jawara. Ceux-ci sont maitres de l'initiation martiale locale, le silat, et ce statut leur a permis de diriger des réseaux qui ont joué un rôle central au cours de différentes périodes de l'histoire de Banten aux côtés des chefs religieux, les kiai. À compter de la dissolution du sultanat de Banten par le gouvernement colonial hollandais en 1813, les kiai et jawara ont développé d'importants réseaux militants anticoloniaux. Ils se sont illustrés dans près d'une vingtaine de rébellions tout au long du $\mathrm{XIX}^{\mathrm{e}}$ siècle, lesquelles culminèrent avec la révolte paysanne anticoloniale de 1888 (documentée par l'historien Sartono Kartodirdjo, 1966). Un autre historien, Michael Charles Williams (1990), a décrit le rôle des kiai dans la direction de larges mouvements de lutte armée, telle la rébellion dite « communiste » de 1926, puis la révolution pour l'indépendance nationale entre 1945 et 1949.

Ancrés au sein de confréries soufies locales tout en participant aux mouvements nationalistes développés à l'échelle du pays, les réseaux militants dirigés par les kiai et les jawara ont été utilisés durant la période sukarnoïste (1945-1967) afin de contrer l'influence du mouvement islamique autonomiste Terre d'islam (Darul Islam, DI) à Java Ouest. Par la suite, certains pans de ces réseaux ont été graduellement contrôlés par le gouvernement du président Suharto, durant le régime appelé Ordre Nouveau (Orde Baru, 1967-1998). Celui-ci a essentiellement appliqué une tactique de division des différents groupes locaux de jawara et a visé à affaiblir la position des kiai au sein des institutions politiques, certains leaders religieux promouvant pour Banten des projets autonomistes, voire séparatistes.

Haji Sochib, alors jawara renommé, et protégé du prestigieux leader religieux Kiai Mahmud, fut désigné pour coopter les chefs religieux et les forces de jawara au sein de deux organisations, respectivement l'Unité fonctionnelle des Oulémas (Satuan Karya Ulama, Satkar Ulama) et l'Unité fonctionnelle des Jawara (Satuan Karya Jawara, Satkar Jawara), fondées en 1971 et 1972. La Satkar Jawara prit par la suite le nom de Pendekar Banten (PB). Le terme pendekar, notamment véhiculé au travers d'épopées semi-légendaires et plus 
récemment par le biais de productions cinématographiques et d'une littérature abondante de bandes dessinées (telle que l'a étudiée Marcel Bonneff 1976), désigne un maître d'arts martiaux, défenseur de valeurs éthiques, incluant la protection des plus faibles et des opprimés. Le terme a été utilisé pour remplacer celui de jawara, alors connoté négativement suite aux campagnes suhartoïstes violentes visant à éradiquer les gangs des grands centres urbains, une partie de la criminalité (Bertrand 2003 : 16), ainsi que les réseaux clientélaires liés aux jawara proches de l'opposition. Sous la supervision de la division militaire Siliwangi, basée à Bandung, et sous la tutelle de l'organe politique du régime, les Groupes fonctionnels (Golongan Karya, Golkar), l'organisation PB maintint la région sous contrôle pendant toute la période de l'Ordre Nouveau.

Sa participation à la répression de la coalition islamique du Parti pour l'unité et le développement (Partai Persatuan Pembangunan, PPP) au cours des élections législatives de 1977 et 1982 conduisit à des dissensions entre Sochib et Embay Mulya Syarif. Celui-ci, considéré comme un «jawara blanc " en raison de ses affiliations au sein des organisations religieuses, choisit alors de développer ses propres affaires dans le secteur de la finance islamique (au travers de la micro-finance et de banques charia) ainsi que du transport de personnes et marchandises entre Jakarta et Lampung. Il continua aussi à entretenir un rapport de patronage avec des représentants politiques et militaires (ces deux catégories étant liées par le biais de la double fonction politique et militaire de l'armée, nommée dwifungsi) au plus haut niveau.

Après la chute du régime du président Suharto en 1998, à l'aube d'une période de réformes appelée Reformasi, un ensemble de jawara et de figures charismatiques du sud bantenois relancèrent le projet d'autonomie régionale qu'ils avaient été forcés de délaisser près d'un demi-siècle plus tôt. Embay Mulya Syarif réactiva alors les réseaux du parti PPP et renforça son activité au sein d'organisations religieuses politisées. En 1998-1999, sa position dans l'Union des intellectuels musulmans d'Indonésie (Cendekiawan Muslim Indonesia, ICMI), dirigée par Bacharuddin Jusuf Habibie - élu peu après président de la République -, lui permit d'être l'un des acteurs principaux de la création des Auxiliaires de sécurité (Pamswakarsa). Cette milice auxiliaire de l'armée était destinée à soutenir la tenue d'une session extraordinaire de l'Assemblée délibérative du peuple (Majelis Permusyawaratan Rakyat, MPR) face aux manifestations étudiantes massives visant à réclamer une réforme en profondeur des institutions politiques et militaires.

Embay Mulya Syarif fut ensuite l'un des principaux initiateurs de la création de la province de Banten, participant en même temps à la formation d'un comité de préparation pour introduire la charia dans le décret d'autonomie. Sochib, qui avait initialement obtenu sa position comme intermédiaire du gouvernement au travers de son opposition à l'autonomie provinciale, se positionna peu avant sa promulgation comme l'un de ses promoteurs. À partir 
de l'accession de Banten au statut de province, en 2000, les réseaux de Sochib se détachèrent partiellement du contrôle central. En outre, avec les réformes relatives à la démocratisation, il renouvela ses stratégies politiques, passant des techniques de coercition physique à des politiques de lobbying visant à capter les votes des électeurs.

Suivant cette logique, Sochib entreprit un recentrement des alliances politiques au niveau familial, au détriment des réseaux clientélaires de jawara. Il reconnaissait officiellement avoir eu vingt-trois enfants (Mansur 2000 : 16) avec six femmes (Tempo 7 octobre 2013) et maintint son mariage avec quatre des femmes qu'il a épousées. Il positionna les enfants issus de ses deux premières épouses à des postes politiques de premier ordre et attribua aux enfants qu'il eut avec ses autres épouses des positions dans l'administration ainsi qu'au sein d'organes économiques charnières du développement régional.

Plusieurs femmes de Sochib ont aussi occupé des positions politiques importantes. Sa jeune femme, Heryani Yuhana, fut soutenue par près d'une dizaine de partis lors des élections départementales de 2011, ce qui lui permit d'accéder à la fonction de vice-préfète de Pandeglang en tant qu'adjointe d'Erwan Kurtubi, l'un des membres historiques du Pendekar Banten.

En outre, la famille de Sochib a investi les parlements de façon massive, notamment à partir de 2009. La législation n'empêche aucunement les représentants de l'autorité au niveau des départements et des villes d'occuper en même temps des sièges dans les assemblées parlementaires. Ainsi, Tatu Chasanah - qui est membre du Conseil de direction du Golkar - fut élue présidente du parlement du département de Pandeglang. La fille de Sochib, Ratu Lilis Karyawati, fut présidente du Conseil régional de direction du Golkar de Serang (avant d'être remplacée par son frère aîné, Chaeri Wardana) et son époux, Aden Abdul Khaliq, est membre du parlement provincial. Deux des épouses de Sochib, Ratna Komalasari et Heryani Yuhana, sont l'une dans le parlement de la ville de Serang, l'autre dans celui du département de Pandeglang. L'époux d'Atut Chosiyah, Hikmat Tomet (décédé en 2013), fut élu membre de l'assemblée nationale aux élections législatives de 2009 grâce à son poste de président du Conseil régional de direction du Golkar. Leur fils, Andika Hazrumy, est membre du parlement national et son épouse, Ade Rossi Chaerunnisa, est membre du parlement de la ville de Serang.

Cet investissement massif des postes et fonctions politiques s'appuie sur un réseau de financement complexe et il participe à l'alimenter.

\section{Les monopoles économiques de la famille de Sochib}

La prospérité économique de la famille d'Atut Chosiyah s'est vu renforcée par les positions acquises par les enfants de la troisième femme de Sochib, Rafiah Suhaemi, au sein de la chambre de commerce et d'industrie provinciale, ainsi que dans les branches de Serang de l'organisation de l'Union des 
entrepreneurs en construction d'Indonésie (Gabungan Pengusaha Konstruksi Indonesia, Gapensi) et de l'Institut de développement des services de construction (Lembaga Pengembangan Jasa Konstruksi,LPJK). À ce titre, la famille a poursuivi la stratégie d'investissement qu'avait entreprise Sochib dès le début de sa carrière entrepreneuriale en misant sur le développement de l'entreprise de construction Sinar Ciomas. Elle n'effectue pratiquement que des projets de construction et de développement physique, comme la construction et la maintenance des routes et autoroutes, le logement, les immeubles, le développement urbain, les services liés à l'irrigation, les services relatifs à la santé, les bureaux des services généraux, ou ceux de l'équipement. En 2003, ce type de projets représentait plus de $51 \%$ du budget pour le développement de la province.

Sochib possédait aussi plusieurs entreprises à travers des prête-noms. En outre, à partir de la création du $\mathrm{PB}$, il percevait $25 \%$ des recettes sur chaque projet réalisé par les entreprises membres de l'organisation (PB $1983: 4$ ). Il investit alors les organes en charge de la diffusion des appels d'offre, procédé qu'il a reproduit à partir de la Reformasi dans le domaine des services, en surévaluant le tarif de ses prestations et en ajoutant à ses projets des taxes additionnelles. Le PB bénéficiait aussi d'importantes commissions sur les recettes d'entreprises extérieures, comme Armada Jaya Perkasa, la plus grosse entreprise de transports quotidiens et touristiques de Banten. Cette entreprise jouit du soutien de Lulu Kaking, allié de Sochib depuis ses débuts et dirigeant de la milice civile ultranationaliste des Jeunesses de la doctrine Pancasila (Pemuda Pancasila).

Par ailleurs, à partir de la Reformasi, la dérégulation de l'industrie manufacturière, du commerce et de l'agriculture ont stimulé les investissements extérieurs, notamment sino-indonésiens. Dans le sud, le secteur de l'huile de palme est largement dominé par Globalindo Agro Lestari, compagnie appartenant au groupe chinois Sin Tek Huat. La gestion de son activité est émaillée par des conflits fonciers avec les paysans locaux. Les contentieux avec les communautés locales touchent aussi l'entreprise Cemindo Gemilang, dont les sites de cimenterie à Bayah et Ciwandan emploient des centaines de travailleurs chinois, en partenariat notamment avec la compagnie China Harbour Indonesia. Ces entreprises prospèrent grâce au soutien politique d'élus locaux et de personnalités au niveau national, comme l'ex-ministre de l'économie, Hatta Rajasa.

Les parts de marché chinoises et sino-indonésiennes sont destinées à se renforcer au cours des prochaines années. Depuis l'élection du président Joko Widodo en juillet 2015, les investissements étrangers ont triplé et ils s'intensifieront probablement suite aux accords récents d'ouverture des frontières de I'ASEAN Economic Community (AEC), en articulation avec celui qui concerne la zone élargie de marchés ouverts du Regional Comprehensive Economic Partnership (RCEP). Par ailleurs, des accords de coopération ont été évoqués lors du sommet de l'APEC en 2014 avec le président chinois $\mathrm{Xi}$ Jinping, dans les domaines de la construction portuaire, ferroviaire et de l'énergie électrique (un projet est déjà en cours à Cilacap). 


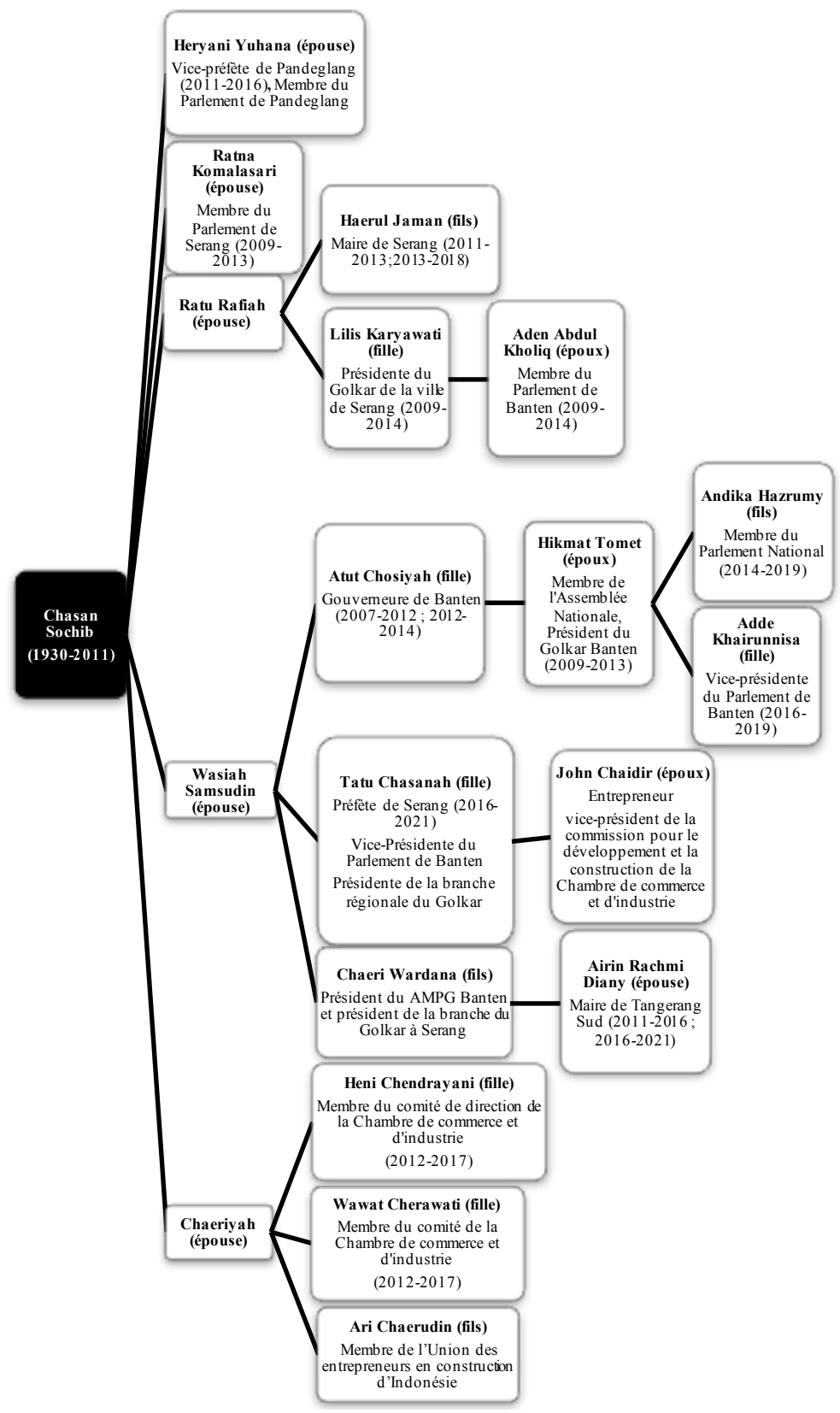

Fig. 1 - Les principaux postes politiques et fonctions économiques occupés par les membres de la famille de Sochib (liste non exhaustive). 
Les mannes liées à la maîtrise du capital privé ainsi que l'habileté des membres du PB à capter des fonds gouvernementaux leur donnent la capacité de financer d'importantes campagnes électorales et de susciter le soutien d'investisseurs influents dans les couches décisionnaires au niveau national. Les succès en politique de la fille aînée de Sochib, Atut Chosiyah, constituent la principale expression publique de cette expansion familiale. Elle occupa d'abord le poste de vice-gouverneure pour la période de 2001 à 2006, suite à une élection qui aurait été marquée par le versement de pots-de-vin et des intimidations à l'encontre de ses concurrents. Elle devint gouverneure en 2005, suite à l'implication du gouverneur alors en poste, Djoko Munandar, dans une affaire de détournement de fonds gouvernementaux destinés à l'aide humanitaire. Sa victoire en tant que gouverneure lors des élections directes en 2006 fut suivie de la nomination de nombreux membres de sa famille à des postes politiques stratégiques, aussi bien au niveau législatif qu'exécutif.

Après les élections provinciales d'octobre 2011, les stratégies népotiques déployées ont suscité des suspicions de financements illégaux (www. humasprotokol.bantenprov.go.id, 8 octobre 2011), conduisant à la formation de plusieurs fronts d'opposition. Néanmoins, les oppositions souffrent d'un manque de résonance dans la société bantenoise. Deux facteurs principaux expliquent la faible visibilité des contre-pouvoirs. Le premier est relatif à la stratégie d'affaiblissement de l'opposition politique menée par la famille de Sochib, notamment au travers d'un jeu complexe de coalitions, d'une fragmentation des unités régionales administratives, puis d'un positionnement des membres de la famille aux postes de direction des institutions politiques ainsi créées. Le second facteur concerne la campagne de communication massive menée par l'équipe de soutien de la gouverneure.

\section{Les arguments philanthropiques d'Atut Chosiyah}

La famille de Sochib a évincé avec succès la plupart de ses concurrents lors des élections des quinze dernières années dans les départements, les municipalités et les parlements. En retour, les montants faramineux qu'elle a captés via les capitaux d'Etat et grâce aux affaires qu'elle a développées servent à financer des campagnes politiques massives qui alimentent ces succès électoraux. Les opérations de communication se manifestent notamment par un affichage intensif de bannières sur les grands axes routiers jusqu'aux localités les plus reculées de la province, et par la distribution de calendriers et posters où figure la photographie des candidats.

Durant les campagnes électorales d'Atut, ces photographies étaient principalement basées sur deux types d'images complémentaires. Le premier présentait la candidate revêtant le costume de gouverneure. Cette mise en image était destinée àl'illustration des événements officiels, commeles manifestations, conventions et réformes politiques. Le second montrait Atut portant le voile 
religieux musulman, dans des mises en scène où elle apparaissait tour à tour comme une figure maternelle des Bantenois, une protectrice attentionnée des paysans et des ouvriers, ou encore une entrepreneuse dynamique et moderne, adepte de musique pop et proche des aspirations de la jeunesse. À travers cette campagne de communication, chaque strate de la société pouvait reconnaitre en Atut une icône et un modèle idéal de société.

La stratégie de marketing des candidats de la famille de Sochib s'accompagne d'un ensemble d'actions de contrôle et de financement d'institutions qui jouent le rôle d'intermédiaires entre le gouvernement et la population. Parmi les principaux organes concernés figurent les Organisations communautaires (Organisasi masyarakat, Ormas), lesquelles rassemblent plusieurs dizaines de milliers de membres dans les sphères de la jeunesse, des associations féminines et de l'humanitaire.

La coordination politique au niveau local est réalisée par l'organisation des Volontaires pour la Banten unifiée (Relawan Banten Bersatu, RBB), un organe destiné à remplacer les pratiques d'intimidation et de coercition physique qui prévalurent sous le régime de l'Ordre Nouveau et au cours des premières années de la Reformasi entre 1999 et 2004. Destinées à assurer les victoires électorales, ces pratiques sont devenues obsolètes suite aux lois nouvelles concernant les élections directes. Le RBB est essentiellement chargé de distribuer des dons matériels et de l'argent aux représentants des villages et d'organiser des événements populaires, comme des concerts, des festivals culinaires, sportifs et artistiques. Cet organe est supervisé par l'Assemblée pour la Banten unifiée (Lembaga Banten Bersatu, LBB), laquelle joue le rôle d'organe de supervision idéologique en cooptant les principales institutions régionales d'information et d'éducation.

L'influence de ces organisations de soutien et de coordination politique s'est considérablement accrue ces dernières années. Elles monopolisent la scène politique au niveau provincial ainsi que dans le département et la ville de Serang. Néanmoins, elles restent en concurrence avec des pôles d'opposition dont les moyens sont plus limités mais qui sont influents dans les départements et municipalités périphériques de Banten.

\section{Les concurrences politiques à partir de la Reformasi (1998)}

À partir de la Reformasi, plusieurs pôles d'opposition au réseau clientélaire développé par Sochib ont fait valoir des revendications concurrentes.

Tout d'abord, au niveau national, la perte de pouvoir politique de l'armée et la réduction de son financement par l'État ont entraîné une privatisation massive du secteur de la sécurité en même temps qu'un accroissement de la concurrence vis-à-vis des réseaux établis à l'époque suhartoïste, comme celui développé par Sochib. Par ailleurs, ce secteur est souvent lié à une économie grise, comprenant le marché du racket organisé ainsi qu'un ensemble d'activités criminelles, 
comme la contrebande, les jeux d'argent illégaux, la traite des personnes, le proxénétisme, le narcotrafic ou encore l'exploitation illégale de ressources naturelles (Wilson $2015: 31$ ).

Ces transformations socio-économiques ont laissé le champ libre aux entrepreneurs de violence concurrents pour investir les secteurs de la criminalité. Ce phénomène a été renforcé du fait que la famille de Sochib a visé à acquérir des positions politiques formelles et à accroître la part de ses activités économiques dans des entreprises légales. Ces orientations se sont traduites par son abandon progressif de l'implication directe dans les activités requérant des méthodes de coercition physique et des actions criminelles.

Nombre d'organisations communautaires se sont alors positionnées comme prestataires pour réaliser des services de sécurité, y compris en faveur de plusieurs associés de Sochib désireux de déléguer ces activités afin de se consacrer à des investissements plus lucratifs et comportant moins de risques judiciaires. Parmi ces prestataires on peut notamment citer le Corps de direction du potentiel de la grande famille de Banten (Badan Pembinaan Potensi Keluarga Banten, BPPKB), fondé par des jawara du centre de la région, à Pandeglang, qui a tissé des réseaux transrégionaux entre Banten et Jakarta (Facal 2012 : 41-42).

En relation à ce phénomène de privatisation de la sécurité, le début de la Reformasi fut aussi marqué par une expansion importante de réseaux criminels sous tutelle de membres de la division régionale des Forces spéciales de l'armée (Komando pasukan khusus, Kopassus). Parallèlement, ces réseaux développèrent une influence politique, laquelle fut marquée par des affrontements directs avec les groupes liés à Sochib. Par la suite, cet essor fut consacré par l'élection de l'ex-colonel des Kopassus, Taufik Nuriman, comme vice-préfet de Serang (2000-2005), puis comme préfet de ce département (2005-2010;2010-2015).

Cette période vit également le renforcement de réseaux d'arts martiaux concurrents, auparavant cantonnés aux activités de sécurité et aux rackets de protection. Ils saisirent l'opportunité offerte par les avancées démocratiques de la Reformasi pour investir la scène politique. C'est le cas de l'école d'arts martiaux Cimande de la région Kebun Djeruk Hilir (Tjimande Tari Kolot Kebun Djeruk Hilir, TTKKDH), dont le secrétaire général, D.A., se présenta en 2008 aux élections municipales de Serang, comme adjoint de l'ancien membre du Pendekar Banten, Jayeng Rana. Malgré leur défaite face à la coalition Khaerul Jaman-Bunyamin, D.A. a conservé son influence politique et occupe les fonctions de chef du Bureau de l'administration publique de la province. Par contre, Jayeng Rana a été écroué en 2015 pour usage de stupéfiants, puis arrêté de nouveau en 2016 pour une affaire de paris illégaux. L'acharnement policier dont il fait l'objet est directement lié à l'hostilité que lui manifeste le Pendekar Banten. Cette opposition est notamment motivée par des conflits 
internes à l'école d'arts martiaux de « la Mère tigre qui roule » (Mande Macan Guling) de la ville de Serang, centrale dès les années 1970 dans les jeux de pouvoir mis en place par le PB (enquêtes personnelles 2007-2016).

L'intensification de la concurrence politique à Banten fut aussi alimentée par les initiatives d'émancipation de proches partenaires de Sochib. Tout en restant sous la tutelle de ce dernier, plusieurs maires et préfets, comme Aat Syafaat, le maire de Cilegon, et Dimyati Natakusumah, le préfet de Pandeglang, créèrent leurs propres réseaux clientélaires, en utilisant eux aussi des pratiques de type népotique et en plaçant les membres de leurs familles à des échelons élevés des instances politiques et administratives. L'introduction du système d'élections directes et l'émergence des partis permettent aux candidats de multiplier leurs affiliations et de mobiliser des mécanismes de coalition de façon tactique, sur le très court terme et en fonction des conjonctures électorales.

Ces stratégies s'appuient aussi sur la mobilisation de groupes de lobbying ainsi que sur le concours de personnalités religieuses. Le renforcement du processus d'éviction systématique des kiai de la scène politique au cours de l'Ordre Nouveau participa directement à constituer une partie de l'élite religieuse comme pôle d'opposition au PB (Hamid 2010 : 422). Cette opposition se manifesta publiquement dès le début de la Reformasi, notamment sous l'égide de l'ancien bras droit de Sochib, Embay Mulya Syarif. Il coopéra tout d'abord avec plusieurs personnalités politiques qui militaient depuis les années 1960 en faveur de l'autonomie régionale, comme Uwes Qorny, dirigeant de deux organisations étudiantes basées à Rangkasbitung, influentes dans le centre et le sud de Banten. Ces militants s'allièrent aussi à diverses personnalités religieuses, tel Hasan Alaydrus, le dirigeant régional de l'organisation islamique conservatrice Muhammadiyah.

Écartés des politiques de développement régional à partir de l'indépendance nationale à cause de leurs visées autonomistes, ces réseaux profitèrent de la Reformasi pour s'organiser et constituer une commission pro-autonomie pour la province de Banten. Ils proposèrent un projet auquel Sochib s'opposa tout d'abord farouchement, puis qu'il récupéra finalement au moment où il fut en passe d'être adopté. Cette récupération s'accompagna de l'éviction des initiateurs du projet, au premier rang desquels Embay Mulya Syarif. Néanmoins, celui-ci continua de financer les campagnes de plusieurs opposants politiques, par exemple celles de Jazuli Juwaini (membre du parlement national pour le parti de la justice prospère [Partai Keadilan Sejahtera, PKS] entre 2009 et 2014) et de Makmun Muzakki (vice-président de la commission $1 \mathrm{du}$ parlement régional pour le parti PPP) lors des élections provinciales de 2012. Muzakki fut par ailleurs l'un des premiers parlementaires à exiger la destitution d'Atut Chosiyah après sa mise en examen en 2013.

Pour contrer ce renouveau de l'islam politique, la famille de Sochib tâcha de mobiliser de nouveau l'organisation islamique de cooptation Satkar Ulama. 
Durant les campagnes électorales d'Atut Chosiyah, le président de cette organisation, Kiai Syahrir Abror, organisa une prière collective de soutien rassemblant près d'un millier d'oulémas (Radar Banten 30 septembre 2006). Enfin, du côté de la famille de Sochib comme dans le camp des différents pôles de l'opposition, s'opéra la mobilisation du soutien de kiai dits « charismatiques ", par le biais du financement de mosquées ou d'écoles coraniques.

Une partie de l'opposition issue de la sphère religieuse fit alliance avec les anciens alliés de Sochib, écartés au début de la Reformasi après que celui-ci eût resserré son noyau clientélaire autour de sa famille. Parmi ces anciens partenaires, on compte notamment Mulyadi Jayabaya, ancien collaborateur de Sochib au sein du PB. Jayabaya parvint à développer des soutiens importants dans la région de Lebak, ce qui lui permit d'être élu préfet de ce département en 2003. Il fut remplacé à la fin de son second mandat en 2013 par sa fille, Iti Octavia Jayabaya, auparavant membre de l'assemblée nationale. Il réussit aussi à positionner sa fille cadette, Diana Jayabaya, comme membre du parlement provincial. Enfin, sa fille benjamine, Mulyanah est membre du parlement de Lebak, tandis que le mari de cette dernière, Agus Wisas, siège au parlement provincial. En 2012, Mulyadi Jayabaya entreprit de former une coalition avec Wahidin Halim, le maire de Tangerang entre 2003 et 2013, en vue de candidater comme vice-gouverneur de Banten.

Wahidin Halim est l'un des principaux rivaux de la famille de Sochib. Il a gravi les échelons politiques en s'appuyant sur un parcours dans la bureaucratie. Aujourd'hui sa famille et son réseau clientélaire ont la mainmise sur les branches exécutive et législative du gouvernement local. Halim bénéficie du soutien de son frère aîné, Nur Hassan Wirajuda, lequel fut ministre des affaires étrangères d'Indonésie entre 2001 et 2009. Lors des dernières élections municipales à Tangerang en 2013, Halim a soutenu son frère cadet, Abdul Syukur, dirigeant de plusieurs branches locales de la milice Pemuda Pancasila, ainsi que membre du parlement de Tangerang entre 1999 et 2009, avant d'intégrer le parlement provincial. L'élection au poste de maire a néanmoins été remportée par Arief Rachadiono Wismansyah, ancien adjoint d'Halim durant ses deux mandats. Enfin, Halim a soutenu son autre frère cadet, Suwandi, pour les élections de 2013 au poste de préfet de Tangerang. Il a toutefois échoué contre Ahmed Zaki Iskandar Zulkarnaen, le fils du préfet sortant, Ismet Iskandar.

La famille de ce dernier constitue l'autre grand pôle d'opposition à la famille de Sochib à Tangerang. Ismet Iskandar fut préfet de Tangerang entre 2003 et 2013 avant d'être remplacé par son fils, Ahmed Zaki Iskandar Zulkarnaen, alors membre de l'Assemblée nationale. La sœur de celui-ci, Intan Nurul Hikmah, est vice-présidente du parlement de Tangerang. Elle s'est présentée lors des élections municipales de 2015, scrutin qu'elle a perdu contre l'épouse de Chaeri Wardana, Airin Rachmi Diany. Quant au fils benjamin d'Ismet 
Iskandar, Ahmed Zulfikar Ibrahim, il est entrepreneur et responsable du club de football de Tangerang, le Persita.

Enfin, en plus de ces différents pôles de rivalité ou d'opposition, le réseau de la famille de Sochib a dû faire face à partir de juin 2011 à la disparition de ce dernier. Bien que cet événement fût anticipé par sa famille - Sochib étant souffrant depuis plusieurs années -, son décès a provoqué des conflits de succession, a remis en question les supports idéologiques mobilisés par les candidats issus de sa famille, et a conduit enfin à un profond renouvellement des stratégies politiques exercées.

\section{Mort de Sochib et reconfiguration des stratégies politiques de sa famille}

Après la mort de Sochib, c'est son fils aîné, Chaeri Wardana, qui a hérité de la charge de gérer les réseaux clientélaires de la famille. Mis en examen à de multiples reprises pour plusieurs cas de corruption et de détournement de fonds, il est notamment accusé d'avoir versé de l'argent à l'ancien président de la Cour constitutionnelle, Akil Mochtar, afin que les voix soient recomptées suite au scrutin qui l'opposa à Iti Octavia lors de l'élection à la préfecture de Pandeglang. Un autre échec majeur des politiques familiales concerne la mise en détention le 20 décembre 2013 d'Atut Chosiyah, alors gouverneure de Banten, notamment accusée d'avoir organisé le détournement de fonds du gouvernement provincial destinés à des équipements de santé.

Ces revers judiciaires indiquent qu'à l'opposé de leur père les héritiers désignés par celui-ci ont échoué a maintenir le contrôle des réseaux clientélaires et des mécanismes politico-économiques locaux qui garantissaient par le passé un ensemble de passe-droits et d'impunités. Ajouté à cela, ces inculpations résultent des moyens nouveaux dont jouit la Commission d'éradication de la corruption (Komisi Pemberantasan Korupsi, KPK) pour mener ses investigations. Néanmoins, malgré ces difficultés, on ne peut sous-estimer la portée des stratégies que Sochib a mises en place au cours des dernières années de sa vie. La façon dont il a positionné les membres de sa famille au sein de postes de contrôle des secteurs économique et politique permet d'assurer les succès électoraux des membres de son réseau clientélaire et de garantir une capacité de financement pour leurs campagnes électorales. De plus, les soutiens locaux que Sochib a mobilisés au travers d'une promotion intensive de l'image de la famille, ainsi que les liens forts qu'il a favorisés avec des représentants politiques au niveau national et au sein des partis, forment un réseau complexe que ses opposants peinent à démanteler.

Par ailleurs, une partie des réseaux de jawara sur lesquels s'est appuyé Sochib dès ses débuts dans le monde de l'entrepreneuriat et en politique reste une base solide de soutien pour la famille (Hamid et Masaaki 2008 : 110). Les écoles de l'initiation martiale silat que dirigent les jawara sont au cour des interactions entre les populations locales, les organisations religieuses et les institutions 
politiques. Elles participent à la coordination des autres groupes de mobilisation politique, comme les organisations pour la jeunesse, les associations pour femmes, ou encore les clubs de vétérans de l'armée.

La persistance de l'influence de ces réseaux d'initiation martiale est visible dans leur mobilisation pour faire pression contre les processus judiciaires engagés contre les membres de la famille de Sochib (cf. Tempo 20 décembre 2013). Ils sont aussi utilisés en amont des différentes élections pour faire la promotion des candidats au sein de leurs localités d'implantation. Dans cette perspective, Atut Chosiyah mit en avant au cours de son mandat de gouverneure son statut de " jawari », l'équivalent féminin de la figure du jawara. La persistance du lien à l'initiation martiale comme socle de légitimité politique transparait aussi dans les entreprises de récupération des groupes d'arts martiaux par les concurrents de Sochib (enquêtes personnelles 2009-2016).

Néanmoins, alors que Sochib avait positionné ces groupes comme des délégataires coercitifs des programmes de contrôle sociopolitique nationaux, leurs porte-paroles mettent désormais en avant leur capacité à représenter les Bantenois dans le cours des dynamiques idéologiques et politicoéconomiques nationales, voire transnationales. Ces auto-attributions se sont révélées récemment lors des campagnes d'opposition à l'expansion régionale de l'organisation État Islamique (EI). En 2015, Embai Mulya Syarif a formé le Front Bantenois anti-ISIS (Front Masyarakat Banten Anti-ISIS, F-MBAI) et la même année le Pendekar Banten a organisé des tribunes publiques et manifestations pour déclarer son hostilité à l'EI.

Cette reconfiguration du rôle des jawara s'ajoute aux ajustements réciproques opérés par les différents concurrents politiques de Banten pour se positionner au sein des dynamiques sociopolitiques régionales et suprarégionales. Ces éléments permettent de formuler des hypothèses concernant la période qui s'annonce suite aux récentes élections provinciales.

\section{Les élections de 2017 : épilogue à la domination de la famille de Sochib ou consécration familiale?}

Les élections provinciales de février 2017 ont donné le ticket HalimHazrumy vainqueur avec un avantage de moins de deux pour cent des voix. Le binôme rival a néanmoins mis au jour des irrégularités dans la campagne électorale des vainqueurs, par exemple lors d'opérations de distribution dans les villages de paquets de nouilles lyophilisées portant les photographies des candidats (Republika.co.id 4 mars 2017). En outre, des preuves concernant l'implication du binôme dans l'achat de voix d'électeurs de la ville de Tangerang et du département de Serang auraient été transmises à la Cour constitutionnelle. Si ces plaintes aboutissaient et si le binôme Rano KarnoEmbay Mulya Syarif l'emportait, on peut s'interroger sur l'impact que cette situation provoquerait sur le politique à Banten. 
Rano Karno est le gouverneur par intérim actuel. En 2007, cet ancien acteur de séries télévisées s'était présenté comme vice-gouverneur aux élections pour le Territoire Spécial de Jakarta, en lice avec Fauzi Bowo, le leader de la milice islamique du Forum de fraternité Betawi (Forum Betawi Rempug, FBR). Rano Karno fut alors impliqué dans une affaire de financements électoraux irréguliers, avant que les charges pesant contre lui soient abandonnées. Plus tard, en 2008, il fut élu vice-préfet pour le département de Tangerang contre Airin Rachmi Diany, l'épouse du fils aîné d'Atut Chosiyah, Chaeri Wardana. Mais en 2011, il mit un terme à son mandat pour siéger comme vice-gouverneur aux côtés d'Atut Chosiyah. Dans le cadre de sa candidature pour les élections provinciales, il a manifesté sa séparation avec les réseaux développés par Sochib, comme l'exprime son rapprochement avec Embay Mulya Syarif, puis le procès qu'il intente au petit-fils de Sochib, Hazrumy.

Pour sa part, l'opposition à l'alliance entre Rano Karno et Embay Mulya Syarif fait ressortir les réseaux clientélaires développés par Wahidin Halim dans la région suburbaine de Jakarta, à Tangerang. Halim bénéficie également de soutiens dans le département périphérique de Lebak, via les réseaux familiaux de l'ex-préfet Mulyadi Jayabaya. Enfin, ces contre-pouvoirs sont renforcés par les collaborations politico-économiques du réseau clientélaire d'Halim avec des investisseurs et des politiciens de niveau national. Ces projets passent notamment par la mobilisation des liens tissés au travers du parti Golkar.

En effet, après une stratégie d'affiliations multipartites durant les premières années de la Reformasi, les membres de la famille de Sochib ont progressivement recentré leurs affiliations sur ce parti. En 2009, l'époux d'Atut Chosiyah, Hikmat Tomet, est devenu président de la branche provinciale du parti. Il a utilisé sa position pour s'assurer que les membres de la famille soient placés en haut des listes de candidature du Golkar dans les différentes circonscriptions électorales. Suite à son décès, en 2013, Tatu Chasanah lui a succédé. Après avoir occupé le poste de vice-préfète durant un mandat à partir de 2010, cette dernière a été élue préfète de Serang en février 2016. Quant à Andika Hazrumy (actuellement membre du parlement national) il a été élu à la place de Khaerul Jaman comme candidat du Golkar pour l'élection de vice-gouverneur. Celui-ci avait préalablement remplacé sa sœur cadette, Lilis Karyawati, comme dirigeant de la branche de Serang du parti.

Ces réseaux n'ont pu contrebalancer le discrédit politique de la famille suite à son implication dans des affaires de corruption, mais celle-ci conserve cependant des positions importantes au sein des instances politiques et administratives au niveau provincial. À Tangerang Sud, la famille jouit du soutien de la maire, Airin Rachmi Diany. Par ailleurs, le soutien d'Irna Narulita à Wahidin Halim, tel qu'il transparut lors des élections provinciales de 2012 via leur alliance face à Rano Karno-Atut Chosiyah est peu probable. En effet, à l'issue de ces élections, l'époux d'Irna Narulita, Achmad Dimyati Natakusumah, a cédé son poste de 
préfet à Erwan Kurtubi, avec pour adjointe Heryani, épouse de Sochib. Or, Irna Narulita a été récemment élue préfète de Pandeglang avec pour adjoint Tanto Warsono Arban, époux d'Andiara Aprilia Hikmat, fille cadette de Atut Chosiyah. Il est donc peu probable que cette alliance au niveau départemental fasse l'objet d'une orientation contradictoire à l'échelle provinciale. Les garanties de stabilité procurées par la famille de Chasan à leurs réseaux d'alliance concernent aussi le réseau familial d'Aat Syafaat à Cilegon.

Forte de ces positions politiques et de ces soutiens, la famille de Sochib est entrée confiante dans sa campagne pour les élections de 2017. Celle-ci a tout d'abord débuté par un rapprochement entre Khaerul Jaman et Rano Karno. Puis Jaman a cédé sa place à Hazrumy. Enfin, celui-ci a rejoint Wahidin Halim. En 2016, le frère cadet de ce dernier, Abdul Syukur, dirigeant de la branche du parti Golkar pour la ville de Tangerang, a publiquement affirmé son soutien à Hazrumy pour les élections provinciales. Puis, Wahidin Halim a lui-même officialisé le rapprochement avec Hazrumy. Il est difficile de connaître les raisons profondes des réorientations d'alliances, mais au vu de ces remaniements il ressort que le facteur idéologique est subalterne par rapport aux arrangements entre partis, entre candidats et, plus largement, entre les réseaux familiaux et de clientèle qui contrôlent les départements et villes de Banten.

\section{Conclusion : une transformation politique incertaine}

Nombre de spécialistes de la Reformasi indonésienne avancent que, même si la décentralisation nationale et le système multipartite ont accru le rôle de la citoyenneté dans la vie politique, ils ont permis en même temps l'intégration d'une partie des réseaux informels du pouvoir dans l'appareil gouvernemental. Vedi Hadiz et Richard Robison (2014 : 54) soulignent par exemple le cas des groupes oligarchiques, lesquels ont renforcé leur institutionnalisation au sein des nouvelles structures démocratiques.

Le cas de Banten montre que, bien que les oligarchies locales aient consolidé et étendu leur champ d'activités, la décentralisation a aussi accru les possibilités de contrôle et de sanctions juridiques. De plus, le processus de démocratisation, bien qu'inabouti, a renforcé les capacités d'opposition au niveau de l'espace public. Cette opposition s'incarne dans des réseaux de contre-pouvoir locaux, tel celui développé dans la région périphérique de Lebak contrôlée par la famille Jayabaya ou encore celui de la zone-tampon de Tangerang, dirigée en partie par la famille Iskandar et par les réseaux clientélaires de Wahidin Halim. Ces réseaux locaux peuvent aussi avoir pour objectif une extension de leur contrôle politique au niveau supra-départemental, comme le montrent l'alliance entre Irna Narulita et Wahidin Halim en 2012, ou encore le soutien apporté à ce dernier par Mulyadi Jayabaya. Ces réseaux profitent de soutiens politiques au niveau national, notamment par le biais de partis, de groupes entrepreneuriaux et de relations clientélaires. 
Cette opposition doit néanmoins faire face à l'hypercentralisation régionale des institutions politiques consécutive à la décentralisation nationale. Elle se combine à la fragmentation des réseaux traditionnels de l'autorité au niveau local -représentés par les réseaux de jawara et les institutions religieuses locales -, exercée à partir de la révolution nationale dès 1949 et plus particulièrement durant l'Ordre Nouveau, via le contrôle sociopolitique délégué à Sochib par le gouvernement du général Suharto. Ces deux processus de fragmentation et d'hypercentralisation politique conduisent à des difficultés d'unification pour les forces de l'opposition. Dans le même temps, ils constituent néanmoins un obstacle au contrôle généralisé du centre, comme le montrent les condamnations récentes de membres de la famille de Sochib, auparavant intouchables.

Cette situation de restrictions réciproques entre anciennes et nouvelles élites fait écho à d'autres contextes régionaux indonésiens actuels, et elle est présente au niveau national. Les élections régionales de Jakarta en février 2017 ont été précédées des mois durant par des manifestations d'une ampleur historique dans la capitale afin de réclamer la destitution du gouverneur, Basuki Tjahaja Purnama. Les résultats du scrutin avec son colistier (42,99 \%), Djarot Saiful Hidayat, ont été serrés au premier tour face à l'ancien ministre de l'éducation et de la culture, Anies Baswedan et son adjoint, Sandiaga Uno (39,95\%). De même, au niveau national le président Joko Widodo, élu en 2014, a été confronté à une forte résistance populaire et à une rivalité des élites, avant sa victoire face à l'ex-général et oligarque, Prabowo Subianto. Cette pression perdure d'ailleurs du fait que le président n'a pas de majorité parlementaire pour gouverner.

Comme à Banten, ces cas montrent toute la difficulté des élites politiques réformistes à s'imposer dans l'arène politique. Ils indiquent également la légitimité dont continuent de jouir les oligarques indonésiens, ainsi que la persistance de la dimension informelle des rapports de pouvoir.

\section{Études}

Bertrand, Romain, « Les virtuoses de la violence. Remarques sur la privatisation du maintien de l'ordre en Indonésie contemporaine », Revue Tiers-Monde, vol. 44, $\mathrm{n}^{\circ}$ 174, 2003, p. 323-344.

Bonneff, Marcel, Les bandes dessinées indonésiennes, Paris, Puyraimond, 1976.

Carnegie, Paul, « Democratization and Decentralization in Post-Soeharto Indonesia: Understanding transition dynamics », Pacific Affairs, vol. 81, n 4, 2009, p. 515-525.

Facal, Gabriel (sous le pseudonyme de Mary van Treche), « Réseaux d'influence et politique locale en Indonésie. Les « hommes forts » de l’organisation Pendekar Banten », Bangkok, Les Carnets de l'IRASEC, n 20, 2012.

Facal, Gabriel, « Hyper-centralization of political power and fragmentation of local authority networks in Banten (Indonesia) since Reformasi », Working Papers, $n^{\circ} 10$, Institute of Asian Studies, Gadong, Universiti Brunei Darussalam, 2014.

Hadiz, Vedi et Robison, Richard, « The political economy of oligarchy and the reorganization of power in Indonesia », in Michele Ford et Thomas Pepinsky (dir.), Beyond Oligarchy: Wealth, Power, and Contemporary Indonesian Politics, Cornell, Ithaca, New York, 2014, p. 35-57. 
Hamid, Abdul et Masaaki, Okamoto, « Jawara in Power 1999-2007 », Indonesia, vol. 86, 2008, p. 109-138.

Hamid, Abdul, "The kiai in Banten: shifting roles in changing times ", in Okamoto Masaaki et Ahmad Suaedy (dir.), Islam in contention: Rethinking Islam and State in Indonesia, Jakarta, Wahid Institute, Kyoto, CSEAS, et Taipei, CAPAS, 2010, pp. 415-438.

Kartodirdjo, Sartono, The Peasants Revolt of Banten in 1888, Gravenhage, Nijhoff, 1966.

Mansur, Khatib, Profil Haji Tubagus Sochib, beserta komentar 100 tokoh masyarakat seputar Pendekar Banten [Profil de Haji Tubagus Sochib, Commentaires de 100 personnalités populaires autour du Pendekar Banten], Cilegon, Pustaka Antara Utama, 2000.

Wilson, Ian Douglas, The politics of protection rackets in post-New Order Indonesia: coercive capital, authority and street politics, Londres, Routledge, 2015.

Williams, Michael Charles, Communism, religion and revolt in Banten, Southeast Asia Series, $\mathrm{n}^{\circ}$ 86, Athens, Ohio, Ohio Center for International Studies, 1990.

\section{Sources}

Persatuan Pendekar Persilatan Seni Budaya Banten Indonesia (PPPSBBI), « Anggaran dasar dan anggaran rumah tangga » [Règlement de base et règlement intérieur], Serang, 1983.

Radar Banten, «Seribu ulama doakan Atut-Masduki » [Mille oulémas prient pour le binôme Atut-Masduki], 30 septembre 2006.

Republika.co.id, " Pilkada Banten Berujung ke Pengadilan MK» [Les élections régionales de Banten s'achèvent en justice à la Cour constitutionnelle], 4 mars 2017 (consulté le 5 mars 2017).

Tempo, « Dukung Atut, Ratusan Jawara Banten Demo di KPK » [Soutien à Atut, des centaines de jawara de Banten manifestent devant les bureaux de la commission anti-corruption], 20 décembre 2013.

Tempo, « Silsilah Dinasti Banten, Abah Chasan dan Para Istri » [La généalogie de la dynastie de Banten, Abah Chasan et ses épouses], 7 octobre 2013.

www.humasprotokol.bantenprov.go.id, "Klarifikasi pemprov Banten tentang klan Sochib kuasai Banten » [Clarification du gouvernement provincial à propos de la mainmise du clan Sochib sur Banten], 8 octobre 2011 (consulté le 25 octobre 2011). 